\title{
Contrasting effects of extended low dose versus standard dose shorter course UFT chemotherapy on microscopic versus macroscopic established tumors: Implications for optimal postoperative adjuvant chemotherapy
}

\author{
JUNJI UCHIDA ${ }^{1}$, HIROYUKI OKABE $^{4}$, KOUSHI NAKANO ${ }^{1}$, AKIO FUJIOKA ${ }^{4}$, HITOSHI SAITO $^{2}$, \\ YOSHIKAZU SUGIMOTO ${ }^{1}$, TOSHINORI OKA ${ }^{1}$ and MASAKAZU FUKUSHIMA ${ }^{3}$ \\ Laboratories of ${ }^{1}$ Optimal Medication Research and ${ }^{2}$ Drug Safety Research, \\ ${ }^{3}$ Tokushima Research Center, Taiho Pharmaceutical Co., Ltd., Tokushima 771-0194; \\ ${ }^{4}$ Advanced Research Laboratory, Taiho Pharmaceutical Co., Ltd., Saitama 358-8527, Japan
}

Received March 26, 2007; Accepted April 30, 2007

\begin{abstract}
Given such differences as relative tumor burden, the optimal dose and schedule for postoperative adjuvant chemotherapy of microscopic disease might be expected to differ significantly from therapy of advanced higher volume disease. We investigated this hypothesis by determining the optimal dose and schedule of the 5-FU pro-drug, UFT, for treatment of early versus later stage disease models of the Lewis lung carcinoma (LLC). Postoperative adjuvant therapy of early stage disease was modeled by intravenous injection of LLC cells and initiating therapy one day later, thus simulating the presence of micrometastases at the time of surgery. As a model of 'late' stage disease, a LLC fragment was implanted subcutaneously and UFT therapy was initiated when the tumor was firmly established and had grown to $>5$ $\mathrm{mm}$ in size. A number of UFT dosing protocols were evaluated such as short-term (daily, for 7 days) maximum tolerated dosing (MTD), e.g. $31 \mathrm{mg} / \mathrm{kg} / \mathrm{day}$, or a much longer-term (e.g., daily, for up to 60 days) repetitive dosing using doses such as $24 \mathrm{mg} / \mathrm{kg} /$ day (the MTD) or lower. The long-term consecutive administration of UFT at relatively low minimally toxic dose levels is a superior dosing regimen in the postoperative adjuvant chemotherapy model; in contrast, the short-term higher dose protocols were superior for treatment of more advanced, established cancer. In addition, the efficacy of UFT in an adjuvant setting is more effective when drug administration is continued for longer periods and
\end{abstract}

Correspondence to: Dr Junji Uchida, Optimal Medication Research Laboratory, Taiho Pharmaceutical Co., Ltd., 224-2, Ebisuno Hiraishi, Kawauchi-cho, Tokushima 771-0194, Japan

E-mail: j-uchida@taiho.co.jp

Key words: uracil and tegafur, postoperative adjuvant chemotherapy, treatment schedule when treatment is initiated at progressively earlier time points, after disease establishment.

\section{Introduction}

Even among cases where the primary tumor has been completely resected or controlled by radical radiotherapy, many cancer patients remain at a high risk of developing recurrent metastatic disease, depending on the clinical stage of the primary lesion. Postoperative recurrence is not uncommon in cases where cancer cells remain that cannot be detected by any imaging modality, or micrometastases have been established before surgery $(1,2)$. Adjuvant chemotherapy is administered to such patients after surgery and/or radiotherapy, with the goal of preventing, or delaying, cancer recurrence and thus prolonging survival of patients.

The efficacy of postoperative adjuvant chemotherapy by 5-FU containing regimens has been demonstrated previously, especially for the treatment for colorectal cancer patients (3). The combination of uracil and tegafur (referred to as UFT) at a molar ratio of $4: 1$ is an oral anticancer agent used for the treatment of both advanced metastatic cancer and, increasingly, for early stage micrometastatic disease. Tegafur is a pro-drug of 5-fluorouracil (5-FU) that is gradually converted to 5-FU in the liver by the cytochrome P-450 enzyme system. Uracil increases the blood concentration of 5-FU by competitive inhibition of dihydropyrimidine dehydrogenase (DPD), the enzyme responsible for 5-FU catabolism. Recently, the efficacy of post operative adjuvant chemotherapy by UFT for non-small cell lung cancer (4), breast cancer (5) and rectal cancer patients (6) has also been reported.

Assessment based on prolongation of survival or improvement of the recurrence-free survival rate is essential for validation of the clinical usefulness of postoperative adjuvant chemotherapy. This necessitates that a large patient sample size, and usually a very long period of follow-up, are required. Moreover, since confirmation of the presence of tumor cells in such situations is difficult, direct analysis of the effect of anti-cancer drugs against tumors is not possible. Thus, 
as far as postoperative adjuvant therapy is concerned, it is extremely difficult to determine the optimal administration schedule for drugs from clinical studies, and experimental approaches are needed to facilitate analyses of this kind.

With this goal in mind, we used a model of metastasis in mice assuming that micrometastases are already present at the time of surgery in patients, and examined the efficacy of UFT for the equivalent of postoperative adjuvant chemotherapy, with the aim of trying to determine the optimal dosage/schedule regimen. In this regard, adjuvant chemotherapy of early stage minimal residual disease frequently involves conventional chemotherapy regimens normally developed and used for treatment of advanced, high volume tumors, including metastases. Because of the toxicity associated with such regimens and the fact that many patients may actually be cancer-free at the time of treatment, it would be useful to determine whether less toxic low(er) dose extended 'tamoxifan-like' schedules using convenient oral drugs taken frequently could have equal or even superior survival/efficacy benefits. Since low-dose frequently administered 'metronomic' chemotherapy regimens are beginning to show efficacy in the clinic even when sometimes treating advanced refractory tumors (7-9), comparing experimentally the effects of such chemotherapy regimens with shorter course conventional maximum tolerated dose (MTD) regimens in settings of early stage microscopic disease versus later stage when there is visible (macroscopic) disease would seem timely.

The purpose of this study was to test the efficacy of various UFT dosing and administration schedules in preclinical settings designed to partially mimic in the clinic early stage microscopic disease versus more advanced established tumors in the clinic. The former (i.e., early stage disease) was modeled by initiating treatment one day after intravenous injection of tumor cells when microscopic metastases, i.e., single cells or small clumps, would be present in the lungs, whereas established subcutaneous tumors or established lung metastases after i.v. injection of the same cells were used as a model of more advanced macroscopic disease. Survival was used as an end-point. Our results suggest that extended daily low-dose metronomic-like administration of UFT is superior to more conventional toxic regimens of the same drug in the setting of the early stage disease model, in contrast to the macroscopic established disease model.

\section{Materials and methods}

Drugs, tumor model and animals. Tegafur (referred to as FT, Taiho Pharmaceutical Co., Ltd.) and uracil (Wako Pure Chemical Industries, Ltd.) were mixed at a molar ratio of $1: 4$, and suspended in $0.5 \%$ sodium carboxymethylcellulose solution. Since FT is the active ingredient of UFT, the amount of only FT was mentioned as the dosage of UFT.

Lewis lung carcinoma (LLC) was maintained by serial implantation of a fragment (2-mm diameter) into the right sub-axially region of male C57BL/6 mice (SLC Japan) every 3-4 weeks. Animals used for experiments were $\mathrm{BDF}_{1}$ (SLC Japan) mice and experiment using animals were carried out in specific pathogen-free (SPF) condition. All experiments were performed in compliance with the regulations of the Animal Experimentation Committee of Taiho Pharmaceutical Co., Ltd.

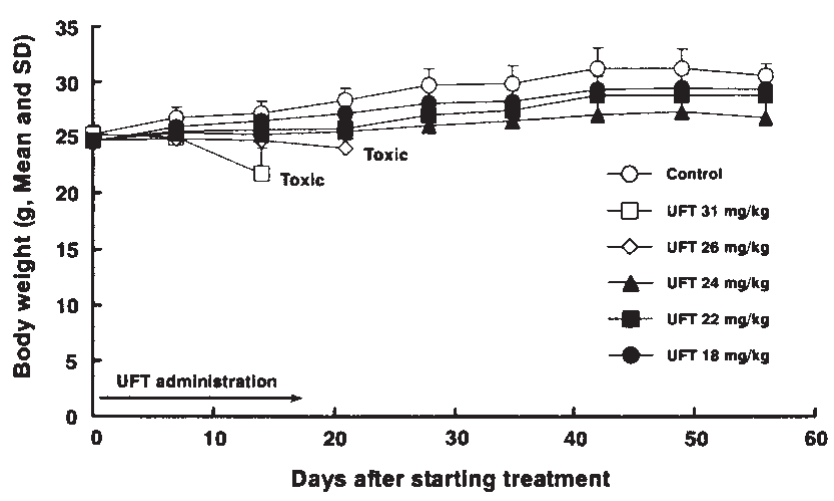

Figure 1. Body weight change of mice by the long-term consecutive administration of UFT. BDF B $_{1}$ mice were orally administered UFT at respective dose level for up to 60 consecutive days in surviving animals. Each point represents the mean body weight and SD of 7 animals. 'Toxic', one or more animals died of toxicity.

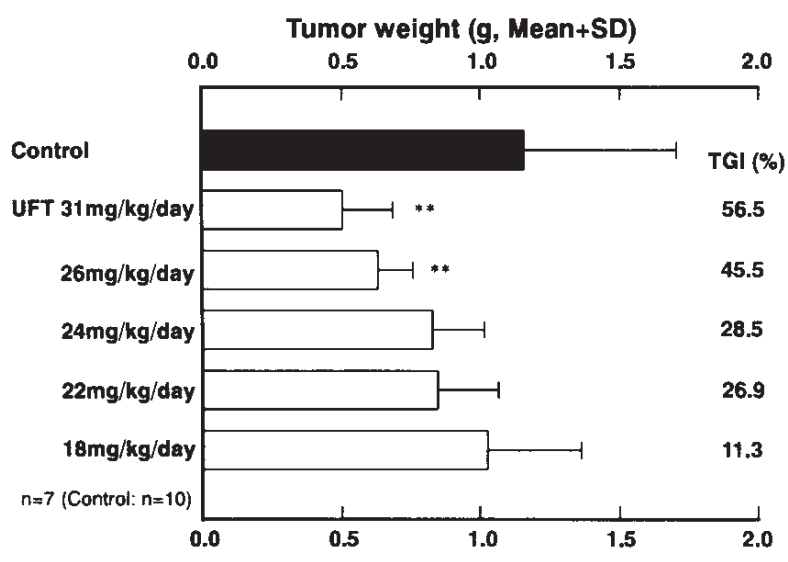

Figure 2. Antitumor effect of UFT in the advanced cancer model established by subcutaneously implantation of LLC. A LLC fragment, 2-mm in diameter, was subcutaneously implanted into the $\mathrm{BDF}_{1}$ mice (day 0 ). UFT was orally administered once daily for 7 consecutive days from day 7 to 13 . On day 14 , the tumor weights were determined. TGI, tumor growth inhibition rate $(\%),{ }^{* *} \mathrm{p}<0.01$ vs. control group (Dunnett's test).

Evaluation of the toxicity of daily oral UFT administration in mice. With the aim of estimating the doses to be used for the efficacy evaluation of UFT, UFT was orally administered once daily to non-tumor-bearing $\mathrm{BDF}_{1}$ mice at the dose of $18,22,24,26$ or $31 \mathrm{mg} / \mathrm{kg} /$ day for up to 60 days in surviving animals. The mice were examined for survival or death, weighed once weekly, and monitored for time-related changes in order to determine the doses to be used for drug efficacy evaluation.

Evaluation of the effect of UFT for intensive treatment in the subcutaneously implanted model with LLC fragments. Solid tumors maintained by serial subcutaneous passage of LLC tumor were removed and fragments $2-\mathrm{mm}$ in diameter were prepared. These fragments were implanted subcutaneously into the right sub-axially region of the $\mathrm{BDF}_{1}$ mice (day 0). Seven days after the implantation, the mice in which the tumor 
Table I. Changes of histopathological findings of pulmonary metastatic regions of mice by the intravenous injection of LLC cells.

\begin{tabular}{ll} 
Time & \multicolumn{1}{c}{ Findings } \\
\hline Day 1 & No remarkable changes \\
Day 2 & Small clusters of tumor cells in the alveolar walls \\
Days 4-5 & $\begin{array}{l}\text { Mild invasive growth of tumor cells } \\
\text { into perivascular areas }\end{array}$ \\
Days 7-10 & $\begin{array}{l}\text { Small or middle nodules of tumor cells } \\
\text { with compression of adjacent tissue }\end{array}$ \\
Day 14 & Middle or large nodules with severe invasion \\
\hline
\end{tabular}

grew to $\sim 5 \mathrm{~mm}$ in diameter were selected and randomized into groups so as to make average body weights uniform among the groups. UFT was orally administered once daily for 7 consecutive days, from days 7 to 13 . On day 14, the tumor weights were determined. The tumor growth inhibition rate (TGI, \%) of each group was calculated using the following formula (1). The drug was regarded as effective when the TGI was $\geq 50 \%$, and the mean tumor weight was significantly lower than that in the control group.

TGI $(\%)=[1-$ (average tumor weight in the treatment group) $/$ (average tumor weight in the control group)] x 100

Histopathological examination of the pulmonary metastasis after intravenous injection of LLC cells. Ascitic fluid specimens containing LLC cells were collected 10 days after the implantation of LLC tumor fragments into the abdominal cavity of C57BL/6 mice. The LLC cells $\left(1.0 \times 10^{4}\right.$ cells) were intravenously injected into the caudal vein of $\mathrm{BDF}_{1}$ mice. One, 2, 4, 5, 7, 10 and 14 days after injection, 3-5 mice were sacrificed on each day and lung tissues were rapidly removed. Each lung tissue was then processed routinely for histopathological examination using conventional H\&E staining.

Evaluation of the effect of UFT in an adjuvant-like treatment setting. LLC cells suspended in physiological saline were implanted into the caudal vein of $\mathrm{BDF}_{1}$ mice at $1.0 \times 10^{4}$ cells per animal (day 0). In the setting for analysis the correlation between treatment schedule (dose level and dosing duration) of UFT and the efficacy on the survival period of mice, UFT administration was initiated on day 1. UFT was orally administered once daily at several dose levels for 7, 14, 28 and 60 consecutive days (administration was continued for up to 60 days in surviving animals).

In the setting for analysis the correlation between starting day of UFT-dosing and the efficacy on the survival period of mice, UFT administration was initiated on day 1, 5, 7 and day 15. UFT was orally administered once daily for 60 consecutive days (administration was continued for up to 60 days in surviving animals) in the fixed dose level at $22 \mathrm{mg} / \mathrm{kg}$ / day.
In both settings, lethality of animals in the control and UFT-treated groups were observed. The efficacy of UFT was evaluated as the elongation of survival period by comparing the median survival time (MST) and 95\% confidence interval $(95 \%$ C.I.).

Statistical analysis. Dunnett's test was applied to assess the significance of differences in the tumor weight between the control group and the UFT-treated groups in the advanced cancer model. The level of significance was set at $5 \%$ on both sides.

\section{Results}

Determination of optimal UFT treatment schedule and dose for adjuvant treatment of low volume vs. higher volume tumors. UFT was orally administered once daily to $\mathrm{BDF}_{1}$ mice at the dose range of $18 \mathrm{mg} / \mathrm{kg} /$ day to $31 \mathrm{mg} / \mathrm{kg} /$ day, and the animals were examined for survival or death. The body weight changes of the animals were monitored weekly. One or more animals died after 2 weeks or more of the drug administration at $31 \mathrm{mg} /$ $\mathrm{kg} /$ day and after 3 weeks or more of the drug administration at $26 \mathrm{mg} / \mathrm{kg} /$ day. Long-term (up to 60 days consecutively) and safe administration of UFT was possible only at doses not exceeding $24 \mathrm{mg} / \mathrm{kg} /$ day (Fig. 1). In the model of advanced cancer, it was expected that a high dose of UFT would be necessary for achieving better anti-tumor effects. Therefore, the 7-day daily administration of the drug that was possible at safe administration of $31 \mathrm{mg} / \mathrm{kg} /$ day UFT was selected as the administration schedule for the advanced cancer model. In the postoperative adjuvant chemotherapy model, a dose not exceeding $24 \mathrm{mg} / \mathrm{kg} /$ day was considered to be more appropriate, as it would allow long-term and safe administration, without inducing any weight loss in the mice. Thus, administration of the drug at $24 \mathrm{mg} / \mathrm{kg} /$ day (MTD in this schedule), $22 \mathrm{mg} / \mathrm{kg} /$ day and $18 \mathrm{mg} / \mathrm{kg} /$ day for 60 days (administration was continued for up to 60 days in surviving animals) was selected. Additionally, 7-day administration at $31 \mathrm{mg} / \mathrm{kg} /$ day and 14-day administration at $26 \mathrm{mg} / \mathrm{kg} /$ day were applied as the short-term and high-dose schedule treatment. In the advanced cancer model, the effective dose level of UFT which showed both $>50 \%$ TGI values and significant differences in tumor weight relative to that in the control group was $31 \mathrm{mg} / \mathrm{kg} / \mathrm{day}$. The anti-tumor effect at doses not exceeding $24 \mathrm{mg} / \mathrm{kg} / \mathrm{day}$, which is the MTD that allows long-term administration with safety, was minimal (Fig. 2).

Efficacy of UFT for adjuvant treatment in the intravenously injected model with LLC cells. The model in which LLC cells were implanted into the caudal vein of mice was used as the postoperative adjuvant chemotherapy, and the effect of the duration or dose level of UFT on the survival-prolonging efficacy was examined. The MST (and 95\% C.I.) in the control group, 7-day administration at $31 \mathrm{mg} / \mathrm{kg} /$ day group, 14-day at $26 \mathrm{mg} / \mathrm{kg} /$ day group, 60-day administration at 24, 22 and $18 \mathrm{mg} / \mathrm{kg} /$ day groups were $26.0(25.0-27.0)$ days, 36.0 (34.0-38.0) days, 38.5 (36.0-39.0) days, 58.5 (56.0-62.0), days 51.0 (48.0-55.0) days and 39.0 (34.0-44.0) days, respectively (Fig. 3). Although survival prolongation was seen in all treated groups as compared to the control group, survival-prolonging 


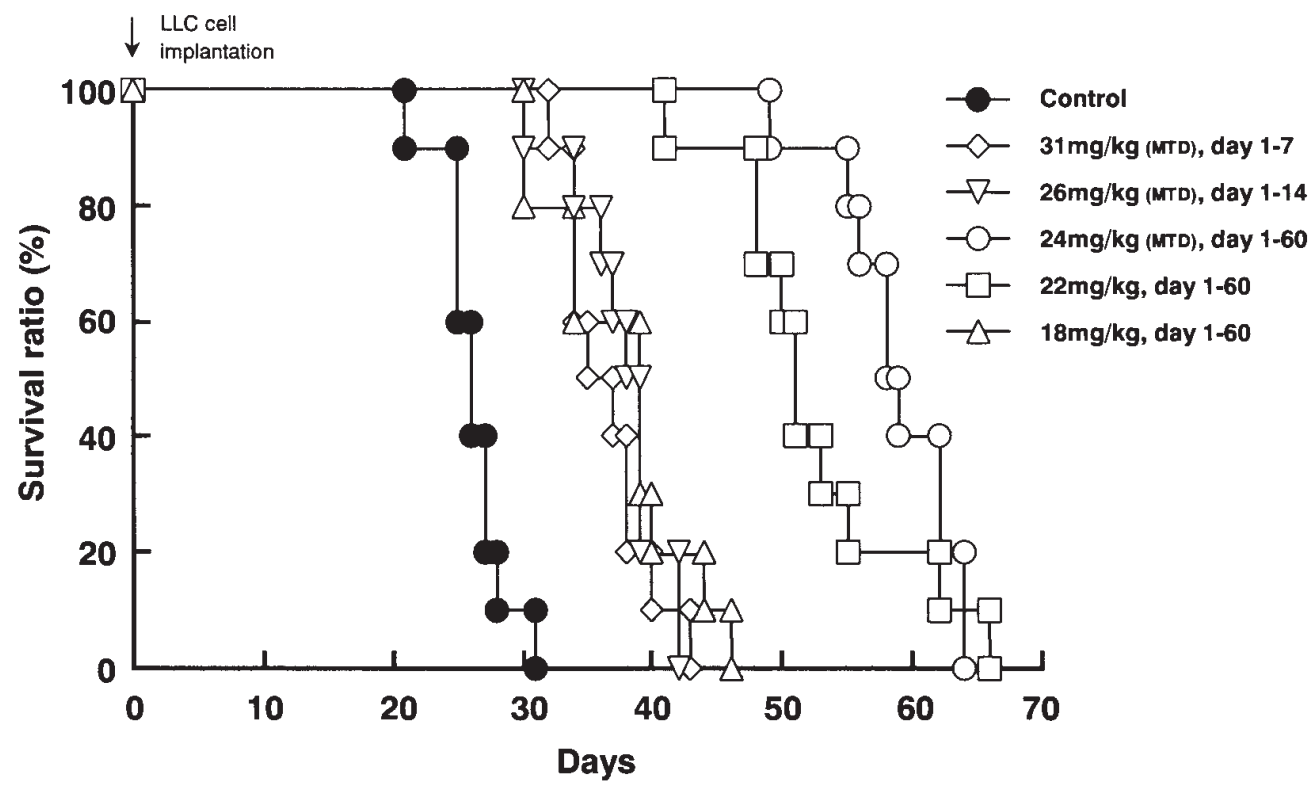

\begin{tabular}{l|c}
\hline & MST (day) (95\% C.I.) \\
\hline Control & $26.0(25.0-27.0)$ \\
\hline UFT $31 \mathrm{mg} / \mathrm{kg}, \mathrm{QD} \times 7$ (day 1-7): MTD & $36.0(34.0-38.0)$ \\
\hline UFT $26 \mathrm{mg} / \mathrm{kg}, \mathrm{QD} \times 14$ (day 1-14): MTD & $38.5(36.0-39.0)$ \\
\hline UFT $24 \mathrm{mg} / \mathrm{kg}, \mathrm{QD} \times 60$ (day 1-60): MTD & $58.5(56.0-62.0)$ \\
\hline UFT $22 \mathrm{mg} / \mathrm{kg}, \mathrm{QD} \times 60$ (day 1-60) & $51.0(48.0-55.0)$ \\
\hline UFT $18 \mathrm{mg} / \mathrm{kg}, \mathrm{QD} \times 60$ (day 1-60) & $39.0(34.0-44.0)$ \\
\hline
\end{tabular}

Figure 3. Antitumor effects of UFT in the adjuvant chemotherapy model established by intravenous implantation of LLC cells. The LLC cells (1.0x10 4 cells/ mouse) was intravenously injected into the caudal vein of $\mathrm{BDF}_{1}$ mice (day 0 ). UFT was orally administered once daily from day 1 according to the respective treatment schedule described above. MST: median survival time, 95\% C.I.: 95\% confidence interval.

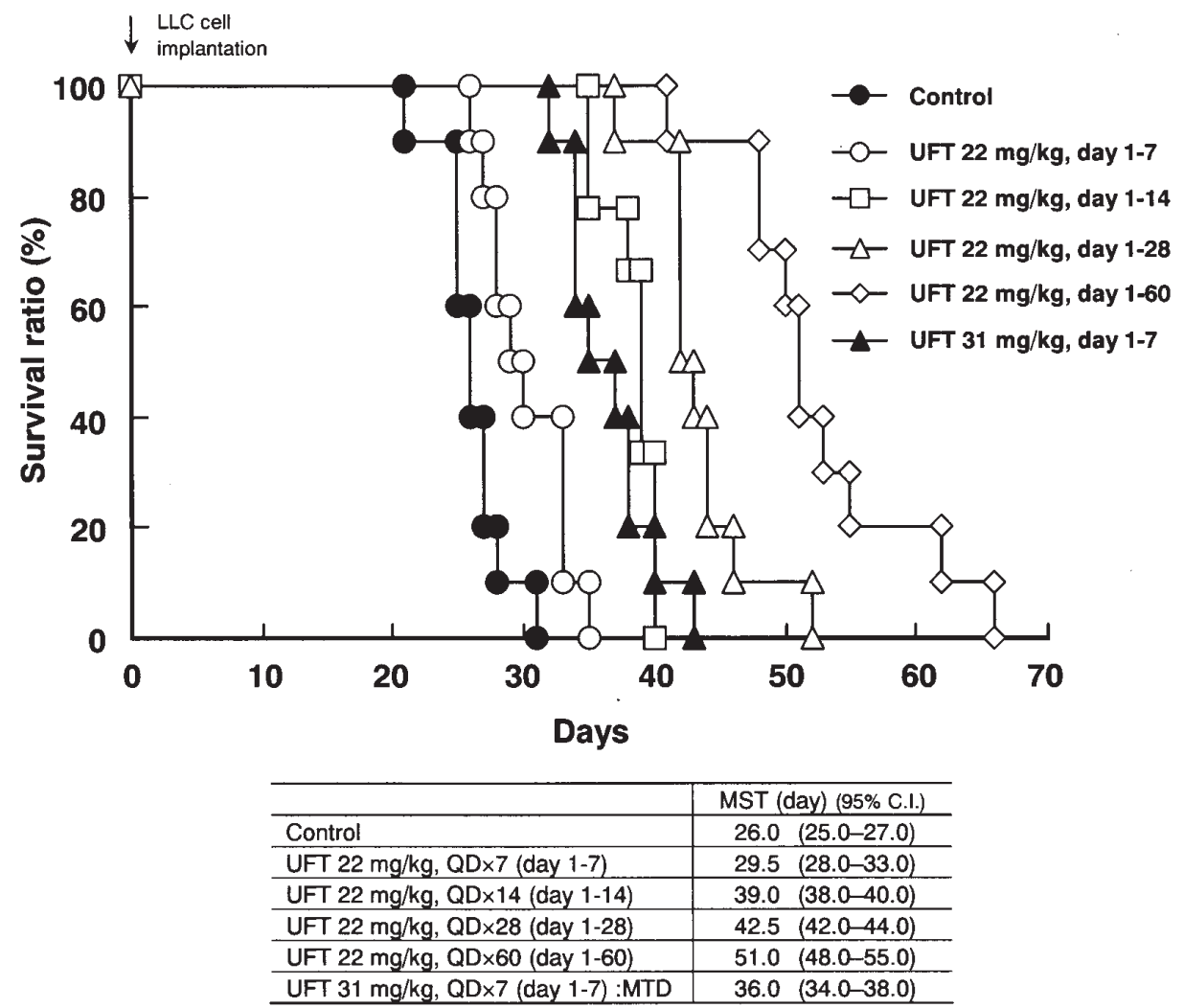

Figure 4. Influence of the treatment duration on the survival-prolonging effect of UFT. The LLC cells $\left(1.0 \times 10^{4}\right.$ cells/mouse) was intravenously injected into the caudal vein of $\mathrm{BDF}_{1}$ mice (day 0). UFT was orally administered once daily from day 1 for 7, 14, 28 and 60 consecutive days in the surviving animals. MST: median survival time, $95 \%$ C.I.: $95 \%$ confidence interval. 


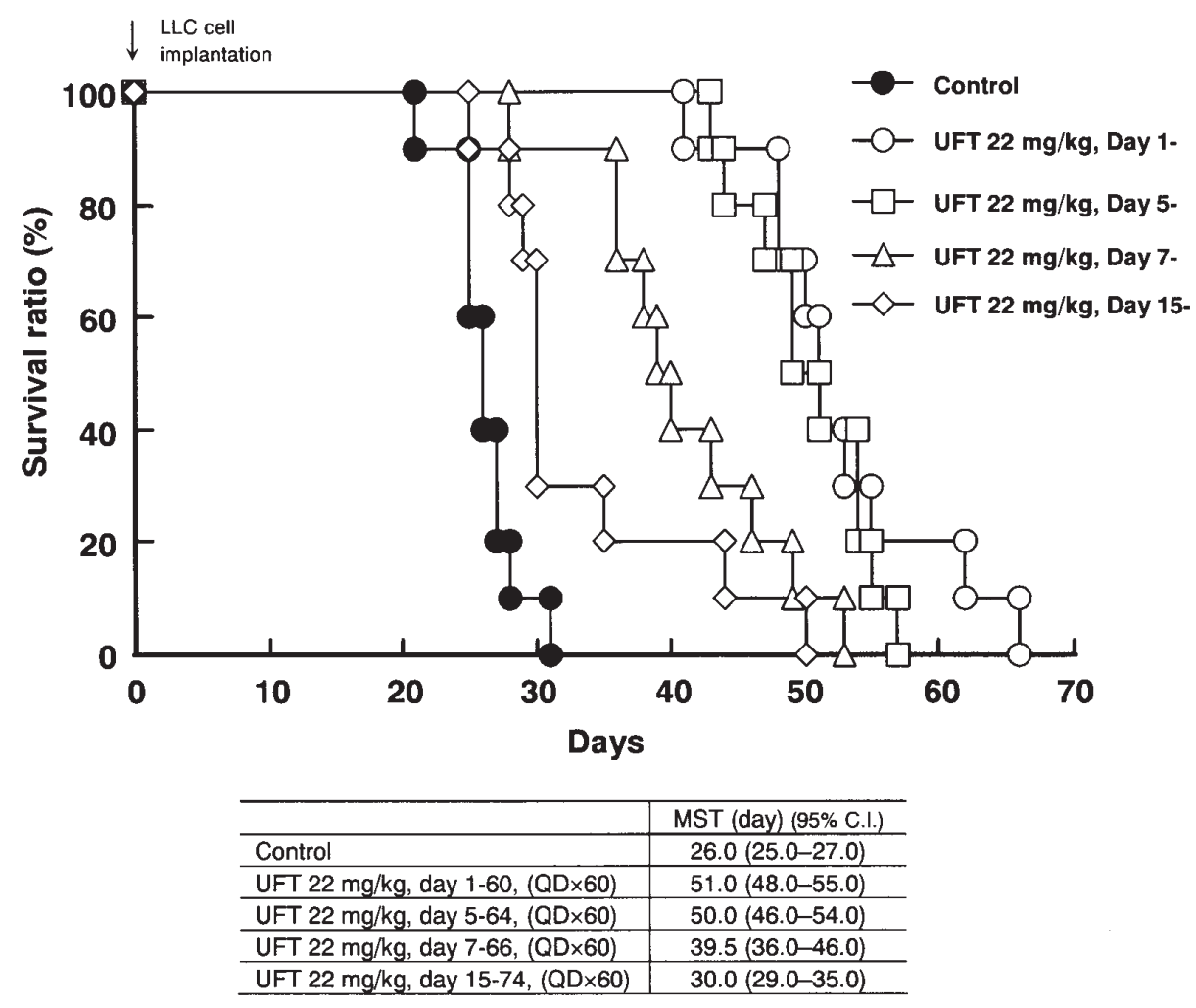

Figure 5. Influence of the start day of administration on the survival-prolonging effect of UFT. The LLC cells $\left(1.0 x 10^{4}\right.$ cells/mouse) was intravenously injected into the caudal vein of $\mathrm{BDF}_{1}$ mice (day 0). UFT administration was started at 1,5,7 and 15 days after implantation and daily treatment was continued for up to 60 consecutive days in surviving animals. MST: median survival time, $95 \%$ C.I.: $95 \%$ confidence interval.

effects by the long-term (60-day) administration at relatively low-dose level (24 and $22 \mathrm{mg} / \mathrm{kg}$ /day) were superior to those of the short-term (7-day or 14-day) administration at highdose level (31 or $26 \mathrm{mg} / \mathrm{kg} /$ day).

Subsequently the effect of the duration of administration on the survival-prolonging effect of UFT was examined. The MST (and 95\% C.I.) in the 7-day, 14-day, 28-day and 60-day consecutive administration groups at fixed dose level $(22 \mathrm{mg} /$ $\mathrm{kg} /$ day) were 29.5 (28.0-33.0) days, 39.0 (38.0-40.0) days, 42.5 (42.0-44.0) days and 51.0 (48.0-55.0) days, respectively (Fig. 4). The survival duration increased with increasing duration of administration of the drug, implying the presence of a correlation between the survival-prolonging effect and the duration of drug administration. The survival-prolonging effect of 28-day and 60-day administration of UFT at $22 \mathrm{mg} / \mathrm{kg} /$ day was even higher than that seen by intensive treatment [7-day administration at $31 \mathrm{mg} / \mathrm{kg} /$ day; MST: 36.0 (34.0-38.0) days].

Changes in the efficacy of UFT in relation to the growth of the metastatic cells. To examine the changes in the anti-tumor efficacy of UFT in relation to the proliferative activity of the tumor cells at the site of metastasis, the starting day of UFT administration after implantation of the LLC cells into the caudal vein of mice was varied, and the differences in the survival-prolonging effect of UFT depending on the starting day of administration was examined (Fig. 5). The MST (and $95 \%$ C.I.) in the control group and the groups in which fixed dose $(22 \mathrm{mg} / \mathrm{kg} /$ day) UFT administration was started on day $1,5,7$ and 15 was $26.0(25.0-27.0)$ days, 51.0 (48.0-55.0) days, 50.0 (46.0-54.0) days, 39.5 (36.0-46.0) days and 30.0 (29.0-35.0) days, respectively. Thus, the survival-prolonging effect decreased as the start of the UFT administration was delayed.

To examine the status of the growth of metastatic lesions on the starting day of administration in each group, pulmonary tissue specimens were examined histopathologically from day 1 to day 14 after the implantation of the LLC cells into the caudal vein of the mice (Table I and Fig. 6). Although the number of LLC cells in each metastatic lesion varied, compression on the adjacent tissues was not observed and the progress of the growth was seen towards the direction of least resistance before day 5 . On day 7, when the survivalprolonging effect was obviously low implying a decrease in sensitivity of the metastatic lesions to the drug, threedimensional growth of metastatic lesions and the apparent compression on the adjacent tissues were observed. On day 14, however, the nodules were found to have grown to a macroscopically visible size.

Thus, a close correlation was observed between the status of the growth of the metastatic lesions on the starting day of administration and sensitivity to UFT.

\section{Discussion}

Our results show that extended daily low-dose chemotherapy regimens (e.g. 28 days), at least when using UFT, appears to be a more effective treatment (as well as being less toxic), when treating low volume (micrometastatic) cancer, whereas shorter courses of therapy using higher drug doses (e.g. 7-14 


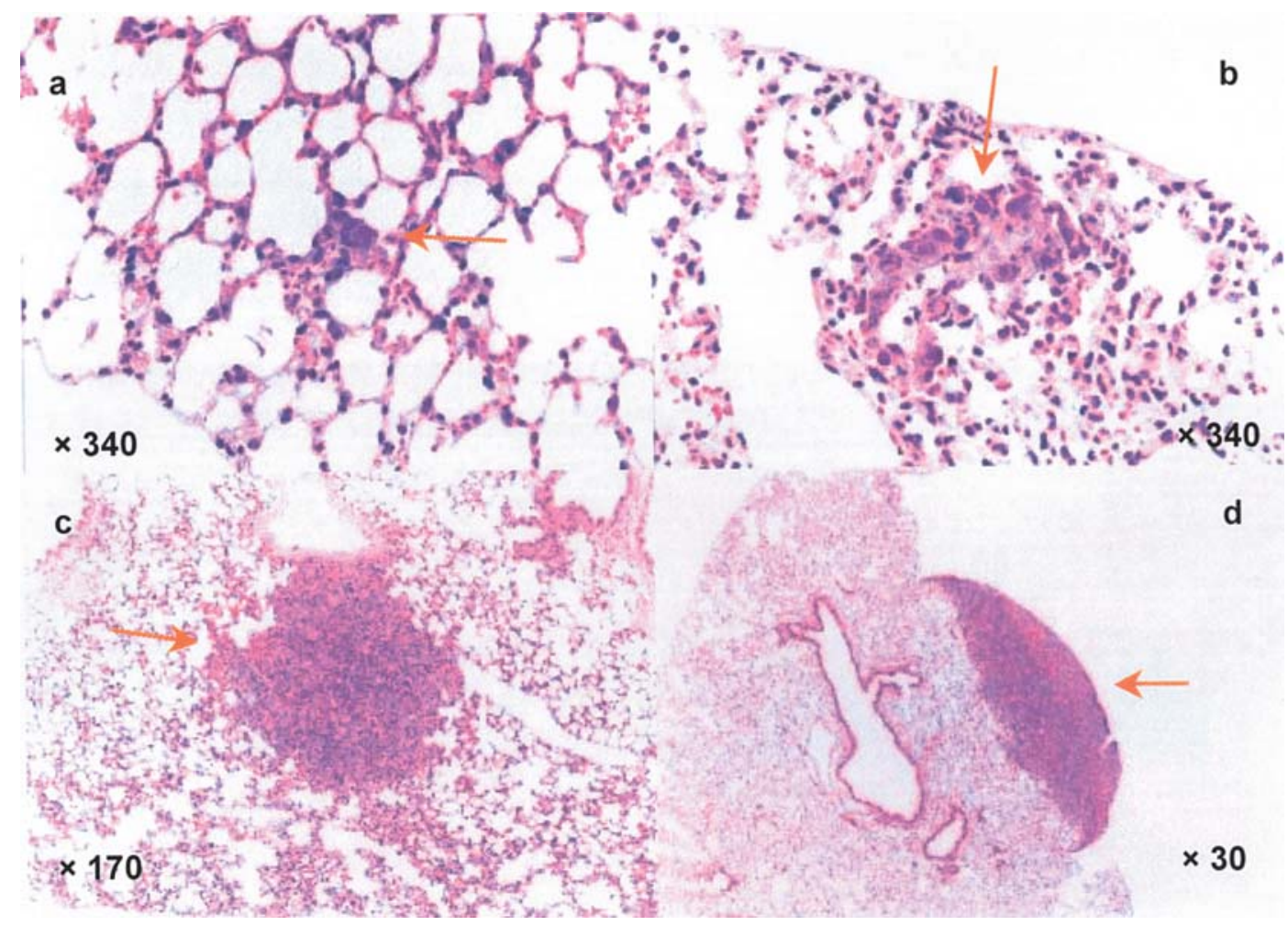

Figure 6. Histopathological findings of the lung of mice after intravenous injection with LLC cells. LLC cells (1.0x10 4 cells/mouse) were injected into the caudal vein of $\mathrm{BDF}_{1}$ mice. Two, 5, 7 and 14 days after injection, 3-5 mice were sacrificed on each day and lung tissues were rapidly removed. Each lung tissue was then processed routinely for histopathological examination using conventional H\&E staining. Each photomicrograph shows the most progressive pattern on 2 (a), 5 (b), 7 (c) and 14 (d) days after injection, respectively. Arrows indicate the tumor cells or nodules.

days), including MTDs, appear superior for control of higher volume, established tumors. In addition, the earlier the treatment is initiated in the micrometastatic disease model, the more effective it is. Our preclinical results may help explain some of the benefits of long-term UFT adjuvant chemotherapy in the clinical setting. For example, meta-analysis of five studies evaluating the efficacy of UFT for postoperative adjuvant chemotherapy in breast cancer revealed improvement of the recurrence-free survival rate after UFT administration at the dose of $300 \mathrm{mg} /$ day or $400 \mathrm{mg}$ /day for 1 or 2 years (10). Even after such prolonged treatment, development of toxicity events greater than grade 2 was not seen. The results of the analysis, stratified according to the duration of UFT administration, revealed that the reduction of recurrence risk was more obvious after administration for 2 years than after 1 year administration of the drug, although the sample size was different. This is similar in principle, to adjuvant tamoxifen treatment for early stage breast cancer where benefits were not seen when using 1 to 2 -year treatments, whereas a survival benefit was detected using much longer-term treatments (11).

In regard to the models used in the present study, the effective dose of UFT in the postoperative adjuvant chemotherapy (minimal residual disease) model was lower than that in the more advanced, established cancer model. This may be because higher levels of the drug were delivered into the smaller lesions, or other factors such as nodule shape may be involved (Fig. 6). However, it is also possible that the surrounding organ/tissue environment, such as the adjacent normal tissues and extracellular matrix, have some influence on the results. For example, a difference in drug sensitivity between metastatic and primary lesions has been reported, based on a comparison of the sensitivity of clinical specimens to the drug (12). Yet another factor is that the extended low-dose UFT chemotherapy treatment represents an example of 'metronomic' chemotherapy, which may inhibit tumor growth primarily by antiangiogenic mechanisms (13). Antiangiogenic treatment strategies are often more effective when used against smaller tumors, presumably by preventing new blood vessel formation; whereas larger tumors would have greater levels of established vessels that are not necessarily sensitive to antiangiogenic drugs. In this regard, UFT has been shown to inhibit tumor angiogenesis, especially when administered by continuous infusion of lower doses of drug compared to bolus weekly injections of higher drug doses (14).

Another important translational aspect of our results is that the combination of a targeted cytostatic agent such as bevacizumab or tamoxifen, with long-term (e.g. 1-2 years, or perhaps even longer) would be possible. Given that such combinations can be effective, even when used for advanced, large volume, tumors $(15,16)$, it might be anticipated that smaller tumor burdens would be particularly responsive. Moreover, short course upfront conventional (higher) dose pulsatile chemotherapy regimens could be followed by longterm maintenance, metronomic type protocols, used in combination with a targeted agent, i.e., 'chemo-switching' (16).

Finally, retrospective analysis of specimens obtained during clinical studies of UFT has shown that better prognosis after UFT treatment is noted in cases where the primary lesions 
had a higher apoptotic index, wild-type p53 expression, and a low expression of dihydropyrimidine dehydrogenase (17). Thus, analysis of the biochemical properties of primary tumors and metastatic cancer cells will also likely be important for the future development of optimal postoperative adjuvant chemotherapy treatments. Some of which may involve long-term low-dose daily administration of chemotherapy drugs such as UFT as we have shown in this report and in clinical studies.

\section{Acknowledgements}

We are most grateful to Professor Robert S. Kerbel at the Sunnybrook and Women's College Health Sciences Centre and the University of Toronto for the detailed review of this study and helpful suggestions.

\section{References}

1. Köhne-Wömpner CH, Schöffski P and Schmoll HJ: Adjuvant therapy for colon adenocarcinoma: Current status of clinical investigation. Ann Oncol 5 (Suppl 3): 97-104, 1994.

2. Kurokawa T, Matsuno Y, Noguchi M, Mizuno S and Shimosato Y: Surgically curable 'early' adenocarcinoma in the periphery of the lung. Am J Surg Pathol 18: 431-438, 1994

3. Moertel CG, Fleming TR, MacDonald JS, et al: Levamisole and fluorouracil for adjuvant therapy of resected colon carcinoma. $\mathrm{N}$ Engl J Med 322: 352-358, 1990.

4. Kato H, Ichinose Y, Ohta M, et al: A randomized trial of adjuvant chemotherapy with uracil-tegafur for adenocarcinoma of the lung. N Engl J Med 350: 1713-1721, 2004.

5. Noguchi S, Koyama H, Uchino J, et al: Postoperative adjuvant therapy with tamoxifen, tegafur plus uracil, or both in women with node-negative breast cancer: A pooled analysis of six randomized controlled trials. J Clin Oncol 23: 2172-2184, 2005.

6. Akasu T, Moriya Y, Ohashi Y, Yoshida S, Shirao K and Kodaira S for the National Surgical Adjuvant Study of Colorectal Cancer: Adjuvant chemotherapy with uracil-tegafur for pathological stage III rectal cancer after mesorectal excision with selective lateral pelvic lymphadenectomy: a multicenter randomized controlled trial. Jpn J Clin Oncol 36: 237-244, 2006.
7. Colleoni M, Rocca A, Sandri MT, et al: Low-dose oral methotrexate and cyclophosphamide in metastatic breast cancer: antitumor activity and correlation with vascular endothelial growth factor levels. Ann Oncol 13: 73-80, 2002.

8. Kieran MW, Turner CD, Rubin JB, et al: A feasibility trial of antiangiogenic (metronomic) chemotherapy in pediatric patients with recurrent or progressive cancer. J Pediatr Hematol Oncol 27: 573-581, 2005.

9. Colleoni M, Orlando L, Sanna G, et al: Metronomic low-dose oral cyclophosphamide and methotrexate plus or minus thalidomide in metastatic breast cancer: antitumor activity and biological effects. Ann Oncol 17: 232-238, 2005.

10. Kasumi F, Yoshimoto M, Uchino J, et al: Meta-analysis of five studies on tegafur plus uracil (UFT) as post-operative adjuvant chemotherapy for breast cancer. Oncology 64: 146-153, 2003.

11. Jordan VC: Tamoxifen: a most unlikely pioneering medicine. Nat Rev Drug Discov 2: 205-213, 2003.

12. Tanigawa N, Mizuno Y, Hashimura T, et al: Comparison of drug sensitivity among tumor cells within a tumor, between primary tumor and metastases, and between different metastases in the human tumor colony-forming assay. Cancer Res 44: 2309-2312, 1984

13. Kerbel RS and Kamen BA: Antiangiogenic basis of metronomic chemotherapy. Nature Rev Cancer 4: 423-436, 2004.

14. Yonekura K, Basaki Y, Chikahisa L, et al: UFT and its metabolites inhibit the angiogenesis induced by murine renal cell carcinoma, as determined by a dorsal air sac assay in mice. Clin Cancer Res 5: 2185-2191, 1999.

15. Klement G, Baruchel S, Rak J, et al: Continuous low-dose therapy with vinblastine and VEGF receptor-2 antibody induces sustained tumor regression without overt toxicity. J Clin Invest 105: R15-R24, 2000.

16. Pietras $\mathrm{K}$ and Hanahan D: A multitargeted, metronomic, and maximum-tolerated dose 'chemo-switch' regimen is antiangiogenic, producing objective responses and survival benefit in a mouse model of cancer. J Clin Oncol 23: 939-952, 2005.

17. Nakagawa T, Tanaka F, Takata T, et al: Predictive value of dihydropyrimidine dehydrogenase expression in tumor tissue, regarding the efficacy of postoperative administered UFT (Tegafur + Uracil) in patients with p-stage I nonsmall-cell lung cancer. J Surg Oncol 81: 87-92, 2002. 